\title{
Molecular Cloning and Tissue Expression of Cell Division Cycle 42 in Buffalo Tissue
}

\author{
${ }^{1}$ Yongchang Hao, ${ }^{2}$ Xiaoru Zhang, ${ }^{1}$ Donglin Zhang, ${ }^{1}$ Ying Cheng, ${ }^{1} \mathrm{Li} \mathrm{Du}$, \\ ${ }^{2}$ Wenhua Kuang, ${ }^{1}$ Ming Lei, ${ }^{1}$ Hanwei Jiao, ${ }^{2}$ Chao Qi and ${ }^{1}$ Fengyang Wang \\ ${ }^{1}$ Hainan Key Lab of Tropical Animal Reproduction and Breeding and Epidemic Disease Research \\ (Construction Period), Animal Genetic Engineering Key Lab of Haikou, College of Agriculture, \\ Hainan University, 570228 Haikou, People's Republic of China \\ ${ }^{2}$ College of Life Science, Huazhong Normal University, 430079 Wuhan, People's Republic of China
}

\begin{abstract}
Brucella sp. are pathogenic bacteria that are internalized by host cells upon activation of cell division cycle $42(\mathrm{Cdc} 42)$ to cause Brucellosis. Buffalo are among the many species that are affected by Brucellosis. The objective of this study was to analyze the expression and distribution of Cdc 42 small GTPases in buffalo which may help elucidating the molecular events leading to Brucella internalization. Full-length buffalo Cdc42 mRNA were obtained and real-time quantitative polymerase chain reaction was performed to assess the transcription level of Cdc42 in heart, liver, spleen, lung, kidney and intestine. Western blot was used to determine the level of Cdc42 in heart, liver, spleen, lung, kidney and intestine tissue; immunohistochemistry techniques were applied for evaluating the distribution of $\mathrm{Cdc} 42$ in tissue. Cdc 42 were found to be expressed in heart, liver, spleen, lung, kidney and intestine tissue and the subcellular localization of Cdc42 was predominantly in the cytoplasma. These data provide important anatomical information on the role of Cdc42 in Brucella internalization in the different buffalo tissue.
\end{abstract}

Key words: Cdc42, real-time quantitative PCR, Western-blot, immunohistochemistry, buffalo, host cell, China

\section{INTRODUCTION}

The Rho (Ras homology) family small GTPase Cdc42 is known to be involved in the regulation of the actin cytoskeletal architecture; the establishment of cell polarity and the regulation of mammalian cell growth (Johnson and Pringle, 1990; Sinha and yaung, 2008; Ridley and Hall, 1992; Nobes and Hall, 1995; Coso et al., 1995). Aberrant activation of $\mathrm{Cdc} 42$ results in pathogenesis such as tumorigenesis and tumor progression, cardiovascular diseases, diabetes and neuronal degenerative diseases (Fritz et al., 2002; Grise et al., 2009; Feng et al., 2011; Stengel and Zheng, 2011).

Brucellae sp. are the causative agent of brucellosis a contagious bacterial disease of animals and a true zoonosis found around the world. Based on its affinity for different hosts, the Brucella genus is composed of six recognized species, B. melitensis, B. suis, B. abortus, $B$. neotomae, B. ovis and B. canis. Despite the fact that the first member of the genus was described $>100$ years ago, the intracellular life cycle and virulence mechanisms of Brucella are not clear, especially for buffalo (Corbel, 1997). The abilities of these bacteria to invade and to survive within cells are decisive factors for causing disease. B. abortus modulates the host cell cytoskeleton to induce its internalization in which $\mathrm{Cdc} 42$ is activated upon cell contact with virulent $B$. abortus but not by a noninvasive isogenic strain (Guzman-Verri et al., 2001).

Current studies on Cdc 42 have mainly focused on its role as a signal transduction convergence point in intracellular signaling networks and its relationship with several pathogenic processes such as Brucella internalization, cancer, cardiovascular diseases and neuronal degenerative diseases. Although, studies describing the expression and distribution of $\mathrm{Cdc} 42$ suggest an important role of $\mathrm{Cdc} 42$ in Brucella internalization in the different tissue of animal including buffalo, there are few reports about its expression in normal tissues.

In a previous study, Cdc42 was found to be primarily located in the Golgi complex (Erickson et al., 1996). In a recent study, comparison of the expression profiles of

Corresponding Author: Fengyang Wang, Hainan Key Lab of Tropical Animal Reproduction and Breeding and Epidemic Disease Research (Construction Period), Animal Genetic Engineering Key Lab of Haikou, College of Agriculture, Hainan University, 570228 Haikou, People's Republic of China 
Cdc42 in human 31 tissues found that $\mathrm{Cdc} 42$ was expressed in almost all tissues were obtained (Schiller, 2006).

To investigate the distribution of $\mathrm{Cdc} 42$ in buffalo tissues, real-time quantitative Polymerase Chain Reaction (PCR), Western blot and immunohistochemistry techniques were performed to assess the expression and distribution of $\mathrm{Cdc} 42$ in heart, liver, spleen, lung, kidney and intestine tissues. The findings suggest that $\mathrm{Cdc} 42$ was ubiquitously present in heart, liver, spleen, lung, kidney and intestine tissues but the expression levels in these tissues are different and provide important information for elucidating the role of $\mathrm{Cdc} 42$ in Brucella internalization in buffalo.

\section{MATERIALS AND METHODS}

Buffalo tissues: Adult Xinglong buffalos, a local buffalo breed were sacrificed and heart, liver, spleen, lung, kidney and intestine tissue were obtained immediately. The tissues used for real time reverse-transcriptase PCR and western blot analyses were initially immersed in liquid nitrogen and preserved at $-70^{\circ} \mathrm{C}$; tissues used for immunohistological analyses were washed with $\mathrm{NaCl}$ $(0.9 \%)$ and fixed with paraformaldehyde (4\%). All animal experimental protocols complied with national legislation and company policy on the care and use of animals according to the related code of practice.

mRNA isolation and Cdc42 full-length cDNA cloning: Total RNA was extracted from Xinglong buffalo tissues as previously described (Sundaresan et al., 2009). For molecular analyses, cDNA synthesis was performed using M-MLV first Strand kit (Invitrogen, USA) and random hexamers with $10 \mathrm{~min}$ incubation at $25^{\circ} \mathrm{C}$ followed $60 \mathrm{~min}$ at $37^{\circ} \mathrm{C}$ and then at $70^{\circ} \mathrm{C}$ for $15 \mathrm{~min}$. Forward primers (F1): 5'-AAAGTGGATACAAAACTGTTTC-3' and Reverse primers(R1): 5'-GCAGAAAGGGCTCTGGAG-3')wereused to perform touchdown PCR reactions as follows: predenaturing at $94^{\circ} \mathrm{C}$ for $3 \mathrm{~min}$; denaturing at $94^{\circ} \mathrm{C}$ for $1 \mathrm{~min}$, annealing at $66^{\circ} \mathrm{C}$ for $1 \mathrm{~min},-2^{\circ} \mathrm{C}$ touchdown at $44^{\circ} \mathrm{C}$ per cycle and extension at $72^{\circ} \mathrm{C}$ for $1 \mathrm{~min}$ for 20 cycles; a final extension at $72^{\circ} \mathrm{C}$ for $5 \mathrm{~min}$. The PCR products were separated by agarose gel electrophoresis. After the PCR products of Cdc42 cDNAs were cloned into pMD20T Vector (TAKARA, Japan) and sequenced.

Real-Time Reverse Transcription PCR (RT-PCR): The PureLink $^{\text {TM }}$ RNA Mini kit (Invitrogen, USA) was used to isolate high-quality total RNA from buffalo tissues according to the manufacturer's instructions. The concentration and integrity of purified RNA were measured by absorption of light at 260 and $280 \mathrm{~nm}$ (A260/280) and electrophoresis after staining with ethidium bromide.

Synthesis of cDNA was performed as described above. Approximately, 100 ng total RNA extracted from the buffalo tissues was used to determine expression levels of Cdc42 mRNA with Platinum SYBR Green qPCR SuperMix-UDG (Invitrogen, USA) using an AB7500 system. The comparative CT method was used to compare the Cdc42 mRNA levels of different tissues. To control variability of RNA input, all PCR reactions were normalized to the Ct value of 28SrRNA of buffalo. Each assay represented three independent experiments and within an experiment, all samples were performed in triplicate. All data are expressed as mean $\pm \mathrm{SD}$. The sequence of the primers for detection of $\mathrm{Cdc} 42$ and 28SrRNA were as follows:

Cdc42-F: 5'-CGCTGAGTTATCCACAAACAGATG-3' Cdc42-R: 5'-GTTATCTCAGGCACCCACTTTTCT-3' 28SrRNA-F: 5'-GTGCTTAGTGGGCTACTTTTGAG-3' 28SrRNA-R: 5'-TGCTGTCTATATCAACCAACACATT TT-3'

Western blot: Western blot analyses were first performed using frozen tissues (approximately $0.1 \mathrm{~g}$ ) ground into powder with a mortar and pestle. To the homogenized tissue, $1 \mathrm{~mL}$ tissue protein extraction reagent (Beyotime, China) and $10 \mu \mathrm{L}$ Phenylmethanesulfonyl Fluoride (PMSF) (1 $\mathrm{mM}$ ) were added to form tissue homogenate. After centrifugation $(12000 \mathrm{~g})$ for $10 \mathrm{~min}$ at $4^{\circ} \mathrm{C}$, the supernatants were obtained and the protein concentrations determined by the Bicinchoninic Acid (BCA) method.

Equal amounts of total protein $50 \mu \mathrm{g}$ were separated by $10 \%$ SDS-PAGE and electrophoretically transferred onto Polyvinylidene Fluoride (PVDF) membrane. Nonspecific-binding sites were blocked with $5 \%$ Bovine Serum Albumin (BSA) for $1 \mathrm{~h}$ and incubated with primary antibodies at $4^{\circ} \mathrm{C}$ overnight.

Primary antibodies consisted of rabbit polyclonal anti-bovine Cdc42 (1:1000) (Boster, China) and anti-rabbit GAPDH monoclonal antibody (1:1200). Secondary antibodies were HRP labeled goat anti-rabbit IgG (1:4000) (Santa Cruz Biotechnology, USA). After incubation with the secondary antibodies for $1 \mathrm{~h}$ at $37^{\circ} \mathrm{C}$, membranes were developed using an ECM kit (Boster, China) for $5 \mathrm{~min}$ and detected by Charge-Coupled Device (CCD) cameras. BandScan 5.0 Software was used to quantify the Western blots for protein expression. 
Immunohistology: Immunohistological analyses were performed with rabbit polyclonal anti-bovine $\mathrm{Cdc} 42$ antibody on buffalo tissue. Briefly, after dehydration and paraffin embedding of tissues, $5 \mu \mathrm{m}$ sections were cut. After slides were dried in an oven $\left(60^{\circ} \mathrm{C}\right)$, paraffin were removed via several changes of xylene and sections were hydrated through a series of graded alcohol concentrations to water and followed by incubation with $3 \%$ hydrogen peroxide for 5-10 min. The tissue sections were blocked with $1 \%$ goat serum for $1 \mathrm{~h}$ to block nonspecific binding sites and treated with rabbit polyclonal anti-bovine $\mathrm{Cdc} 42$ antibody (1:100 dilution) for $1 \mathrm{~h}$ at $37^{\circ} \mathrm{C}$. After washing with Phosphate Buffered Saline (PBS) three times ( $3 \mathrm{~min}$ each), horseradish peroxidaseconjugated goat anti-rabbit IgG-HRP were added to sections and incubated for $30 \mathrm{~min}$ at $37^{\circ} \mathrm{C}$. Color was developed using a color developing kit (Boster, China). Slides were counterstained with hematoxylin (Beyotime, China) before mounting. As a negative control, normal rabbit serum or PBS alone were used in place of rabbit polyclonal anti-bovine $\mathrm{Cdc} 42$ antibody antibody. Sections were observed by microscope at $200 \mathrm{x}$ magnification.

\section{RESULTS AND DISCUSSION}

Cdc42 is a molecule switch involved in multiple cell signaling pathways. There appears to be a close relationship between the specific distribution of $\mathrm{Cdc} 42$ and its biological function (Hussain et al., 2001). To elucidate the relation in the report, firstly, the complete sequences of buffalo $\mathrm{Cdc} 42 \mathrm{cDNA}$ were cloned and deposited into GeneBank (Accession No. JN380079).

Analysis and identification of molecules in the host cells in different tissues involved in the invasion process and trafficking are necessary to understand the events leading to the establishment of the infection. To determine the expression level of $\mathrm{Cdc} 42$ in all buffalo tissues examined and provide important anatomical information on the role of $\mathrm{Cdc} 42$ in Brucella internalization in the different buffalo tissue, real-time RT-PCR, Western blot and immunohistological analyses were performed. The real-time RT-PCR analysis results indicated that $\mathrm{Cdc} 42$ mRNA were ubiquitously present in all buffalo tissues examined. Further the mRNA level observed are listed as follows from high to low: lung, spleen, kidney, heart, liver and intestine (Fig. 1). Similarly, Western blot analyses indicated that the $\mathrm{Cdc} 42$ protein were expressed in all buffalo tissues examined and the quantification data of protein expression indicated that the protein level

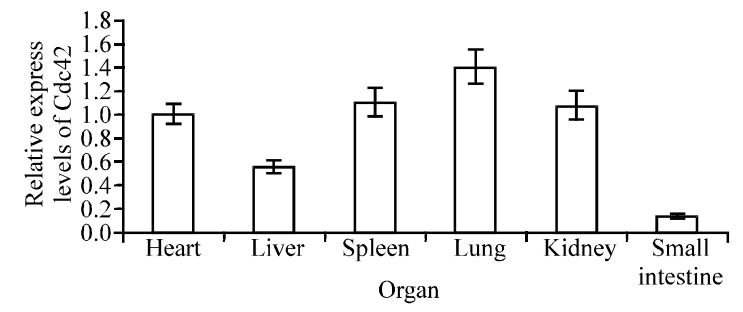

Fig. 1: Relative expression levels of Cdc 42 mRNA in buffalo heart, liver, lung, spleen, kidney and small intestine tissues

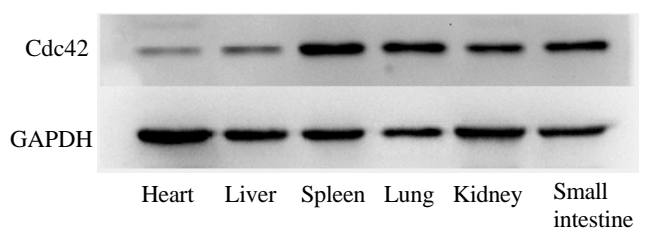

Fig. 2: Western blot analysis of $\mathrm{Cdc} 42$ protein expression in buffalo heart, liver, lung, spleen, kidney and small intestine tissues

from high to low was as follows: lung, spleen, intestine, kidney, liver and heart (Fig. 2). The results of immunohistological analyses with rabbit polyclonal antibovine $\mathrm{Cdc} 42$ indicated that $\mathrm{Cdc} 42$ protein was mainly present in the cytoplasm of cells where weak positive signals were observed in heart and liver and strong positive signals were observed in spleen, lung and intestine (Fig. 3).

Brucella organisms are intracellular parasites of mammals including buffalo. In vivo, Brucella has been described within bovine, caprine and murine trophoblasts in caprine lymphocytes, $M$ cells, chicken embryo fibroblasts as well as a number of professional phagocytes lining different tissues (Holland and Picektt, 1956; Richardson and Holt, 1964; Anderson and Cheville, 1986; Anderson et al., 1986; Ackermann et al., 1988; Detilleux et al., 1988; Cheville et al., 1992, 1996).

Virulent Brucella but not defective Bvr mutants, selectively activates $\mathrm{Cdc} 42$ reaching a maximum of accumulated GTP-loaded Cdc42, $30 \mathrm{~min}$ after bacterial contact with cells. Moreover, B. abortus is internalised more efficiently after intoxication of cells with the Cytotoxic Necrotizing Factor (CNF) which activates Rho, Rac and $\mathrm{Cdc} 42$ and induces membrane ruffles and stress fibre formation (Guzman-Verri et al., 2001).

The spleen is the principal site of infection in mice and analysis of splenocytes following infection of $\mathrm{CD} 1$ mice confirmed the production of $\mathrm{IFN}-\gamma$ protein 


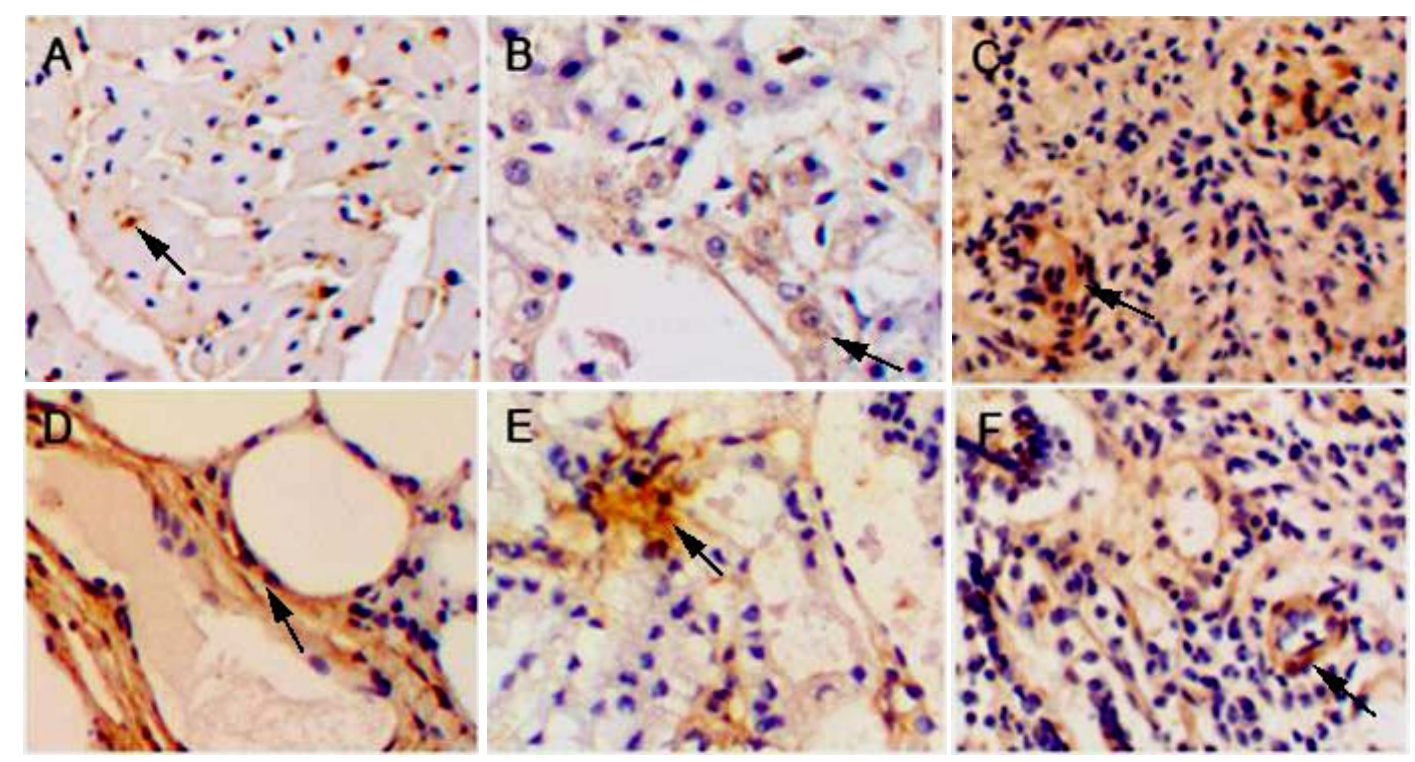

Fig. 3: Distribution of Cdc42 protein in buffalo A) heart, B) liver, C) spleen, D) lung, E) kidney and F) intestine tissue sections. Magnification x200. Arrow indicated Cdc42 protein was mainly present in the cytoplasm of cells

in vivo following $B$. abortus strain 2308 infection (Fernandez-Lago et al., 1996). The study showed that Cdc42 mRNA and protein are ubiquitously present in all buffalo tissues examined with relatively high levels in spleen and lung. These data suggested that it is possible that $\mathrm{Cdc} 42$ may play more important function in spleen and lung than that in other tissues examined.

Cdc42 mRNA and protein levels in kidney, liver, heart and intestine were different indicating that the regulation of expression in these tissues is altered and likely to be correlated with its role in Brucella internalization. The results of immunohistological analyses indicated that Cdc42 protein was mainly present in the cytoplasm which agrees with the observation by Erickson et al. (1996).

\section{CONCLUSION}

The findings provide important information on the distribution and expression of $\mathrm{Cdc} 42$ for its role in Brucella internalization in buffalo. Further studies are likely to include investigating the interaction of $\mathrm{Cdc} 42$ in buffalo tissues with important Brucella bacterial proteins.

\section{ACKNOWLEDGEMENTS}

This study was financially supported by the Major National Science and Technology Specific Projects (2009ZX08007-009B) and the National High Technology
Research and Development Program of China (2011AA100302). Yongchang Hao, Xiaoru Zhang and Donglin Zhang contributed equally to this research.

\section{REFERENCES}

Ackermann, M.R., N.F. Cheville and B.L. Deyoe, 1988. Bovine ileal dome lymphoepithelial cells: Endocytosis and transport of Brucella abortus strain 19. Vet. Pathol., 25: 28-35.

Anderson, T.D. and N.F. Cheville, 1986. Ultrastructural morphometric analysis of Brucella abortus-infected trophoblasts in experimental placentitis. Bacterial replication occurs in rough endoplamic reticulum. Am. J. Pathol., 124: 226-237.

Anderson, T.D., N.F. Cheville and V.P. Meador, 1986. Pathogenisis of placentitis in the goat inoculated with Brucella abortus II. Ultrastructural studies. Vet. Pathol., 23: 227-239.

Cheville, N.F., A.E. Jensen, S.M. Halling, F.M. Tatum and D.C. Morfitt et al., 1992. Bacterial survival lymph node changes, and immunologic responses of cattle vaccinated with standard and mutant strains of Brucella abortus. Am. J. Vet. Res., 53: $1881-1888$.

Cheville, N.F., S.C. Olsen, A.E. Jensen, M.G. Stevens and A.M. Florance et al., 1996. Bacterial persistence and immunity in goats vaccinated with a purE deletion mutant or the parental $16 \mathrm{M}$ strain of Brucella melitensis. Infect. Immun., 64: 2431-2439. 
Corbel, M.J., 1997. Brucellosis: An overview. Emerg. Infect. Dis., 3: 213-221.

Coso, O.A., M. Chiariello, J.C. Yu, H. Teramoto and P. Crespo et al., 1995. The small GTP-binding proteins $\mathrm{Racl}$ and $\mathrm{Cdc} 42$ regulate the activity of the JNK/SAPK signaling pathway. Cell, 81: 1137-1146.

Detilleux, P.G., N.F. Cheville and B.L. Deyoe, 1988. Pathogenesis of Brucella abortus in chicken embryos. Vet. Pathol., 25: 138-146.

Erickson, J.W., C. Zhang, R.A. Kahn, T. Evans and R.A. Cerione, 1996. Mammalian Cdc42 is a brefeldin A-sensitive component of the golgi apparatus. J. Biol. Chem., 271: 26850-26854.

Feng, J.G., Q. Liu, X. Qin, Y.H. Geng and S.T. Zheng et al., 2011. Clinicopathological pattern and Annexin A2 and $\mathrm{Cdc} 42$ status in patients presenting with differentiation and lymphnode metastasis of esophageal squamous cell carcinomas. Mol. Biol. Rep., 39: 1267-1274.

Fernandez-Lago, L., M. Monte and A. Chordi, 1996. Endogenous gamma interferon and interleukin-10 in Brucella abortus 2308 infection in mice. FEMS Immunol. Med. Microbiol., 15: 109-114.

Fritz, G., C. Brachetti, F. Bahlmann, M. Schmidt and B. Kaina, 2002. Rho GTPases in human breast tumors: Expression and mutation analyses and correlation with clinical parameters. Br. J. Cancer, 87: 635-644.

Grise, F., A. Bidaud and V. Moreau, 2009. Rho GTPases in hepatocellular carcinoma. Biochim. Biophys. Acta, 1795: 137-151.

Guzman-Verri, C., E. Chaves-Olarte, C. vonEichel-Streiber, I. Lopez-Goni and M. Thelestam et al., 2001. GTPases of the rho subfamily are required for Brucella abortus internalization in non-professional phagocytes: Direct activation of CDC42. J. Biol. Chem., 276: 44435-44443.
Holland, J.J. and M.J. Picektt, 1956. Intracellular behavior of Brucella variants in chick embryo cells in tissue culture. Proc. Soc. Exp. Biol. Med., 93: 476-479.

Hussain, N.K., S. Jenna, M. Glogauer, C.C. Quinn and S. Wasiak et al., 2001. Endocytic protein intersectin-1 regulates actin assembly via $\mathrm{Cdc} 42$ and N-WASP. Nat. Cell Biol., 3: 927-932.

Johnson, D.I. and J.R. Pringle, 1990. Molecular characterization of CDC42, a Saccharomyces cerevisiae gene involved in the development of cell polarity. J. Cell Biol., 111: 143-152.

Nobes, C.D. and A. Hall, 1995. Rho, rac and cdc42 GTPases regulate the assembly of multimolecular focal complexes associated with actin stress fibers, lamellipodia and filopodia. Cell, 81: 53-62.

Richardson, M. and J.N. Holt, 1964. Multiplication of Brucella in cultured lymphoid and non-lymphoid cells. J. Bacteriol., 88: 1163-1168.

Ridley, A.J. and A. Hall, 1992. The small GTP-binding protein tho regulates the assembly of focal adhesions and actin stress fibers in response to growth factors. Cell, 70: 389-399.

Schiller, M.R., 2006. Coupling receptor tyrosine kinases to Rho GTPases--GEFs what's the link. Cell Signal, 18: 1834-1843.

Sinha, S. and W. Yang, 2008. Cellular signaling for activation of Rho GTPase Cdc42. Cell. Signaling, 20: 1927-1934.

Stengel, K. and Y. Zheng, 2011. Cdc42 in oncogenic transformation, invasion and tumorigenesis. Cell Signal, 23: 1415-1423.

Sundaresan, N.R., M.D. Marcus Leo, J. Subramani, D. Anish and M. Sudhagar et al., 2009. Expression analysis of melatonin receptor subtypes in the ovary of domestic chicken. Vet. Res. Commun., 33: 49-56. 